\title{
WILEY-VCH
}

DOI: 10.1002/((please add manuscript number))

Article type: Communication

\section{Generation of Novel Hybrid Aptamer-Molecularly Imprinted Polymeric Nanoparticles (AptaMIP NPs)}

Alessandro Poma, * Heli Brahmbhatt, Hannah M. Pendergraff, Jonathan K. Watts, and Nicholas W. Turner*

Dr. A. Poma, Ms. H. Brahmbhatt, Dr. N. W. Turner

Department of Life, Health and Chemical Sciences, The Open University, Milton Keynes, MK7 6AA, United Kingdom

E-mail: nicholas.turner@open.ac.uk; alessandro.poma@open.ac.uk

Dr. J. K. Watts, Ms H. M. Pendergraff

Department of Chemistry, University of Southampton, Highfield, Southampton, SO17 1BJ, United Kingdom

Keywords: molecularly imprinted polymers, nanoparticles, aptamers, solid-phase synthesis, cocaine

Molecular recognition processes are at the basis of the development of diagnostic devices and applieations-as well as drug-receptor, antigen-antibody and many more similar interactions. For many years the dominance of antibodies has remained unchallenged for analytical, diagnostic and therapeutic applications. ${ }^{[1]}$ Nonetheless, antibodies have disadvantages including elevated cost, immunogenicity, and poor shelf-life and stability, ${ }^{[2]}$ and much research is directed toward the development of suitable alternatives. ${ }^{[3]}$

Several alternatives have already been developed based on diverse materials. ${ }^{[3-4]}$ For example, given the protein nature of antibodies, engineered binding proteins (EBPs) and fragments have been produced. ${ }^{[5]}$ Nonetheless, despite their lower cost in comparison with antibodies, the stability of EBPs and their poor integration with sensing platforms still remain problematic.

Another promising alternative material is nucleic acids: aptamers (from the Latin aptus - fit, and Greek meros - part) are oligonucleotides that bind to a specific target molecule and have started to represent more and more a suitable alternative to antibodies, since they can be manufactured on both small and large scale by automatic processes, which makes them 


\section{WILEY-VCH}

reasonably cost-effective. ${ }^{[6]}$ In addition, they are more intrinsically stable than antibodies to environmental conditions (denaturation of a double-strand of DNA usually needs a temperature up to $\left.95{ }^{\circ} \mathrm{C}\right) .{ }^{[7]}$ Nonetheless, they tend to be susceptible to degradation by ubiquitous nuclease enzymes, ${ }^{[8]}$ and their technology, until recently, was well covered by intellectual property, thus making it poorly suitable for large-scale application and evaluation. ${ }^{[3,9]}$

A third alternative to antibodies, probably not as well-known as the previous two, is represented by molecularly imprinted polymers (MIPs). ${ }^{[10]}$ The last thirty years have seen a dramatic increase in the research and applications of these materials. ${ }^{[11]}$ MIPs are fullysynthetic polymeric materials produced around the target molecule (template); after the polymerization process, the template is washed away from the material, leaving behind cavities suitable to recognize and rebind the molecule again. They can be selective for both shape and chemical functionality. ${ }^{[10]}$ Nonetheless, so far MIPs have promised much more than they have actually delivered, especially in terms of application, since their rebinding performance is often inferior to that of antibodies or aptamers. ${ }^{[3]}$

A better synthetic substitute might be represented by MIP nanoparticles (MIP NPs) ${ }^{[12]}$ which better mimic their natural protein counterparts, not only in terms of rebinding performance but also regarding their size and dispersibility-characteristics. ${ }^{[13]}$ Nonetheless, even if these nanomaterials are more eomparable and suitable to mimic the actual antibody proteins, their composition is still based on the same "classic" monomers which have been used for the past 30 years to produce bulk MIPs, definitely not tailored for the specific target molecule. ${ }^{[10]}$ Strategies like combinatorial synthetic approaches or computational modelling have advanced and improved the situation significantly, but a lot remains to be done. ${ }^{[14]}$

A potential promising system might be a hybrid which integrates two or more of the technologies described above. ${ }^{[15,16]}$ The group of Allender et al. has already looked into introducing short peptide-sequences into polymer matrixes in order to confer increased 


\section{WILEY-VCH}

specificity and selectivity deriving from-thanks to the chemical complexity of the proteinderived recognition element. ${ }^{[15 b]}$ This approach, despite being very elegant and promising, has been very difficult to optimize successfully.

In our opinion, a better option might be represented by a new category of imprinted materials which integrate more resistant and versatile aptamer monomers inside their structure. A first attempt at this has been explored by Spivak et al., who have introduced aptamer strands into molecular imprinted hydrogels for proteins (thrombin, PDGF- $\beta \beta$ ) or viruses-particles, ${ }^{[16]}$ which resulted in the production of materials that were able to selectively swell (or shrink) in the absence (or presence) of the template. However, in this work the introduction of the aptamer monomer was performed by modifying the aptamer sequences using 5'-phosphate Acrydite $^{\mathrm{TM}}$ modification, which leaves the DNA strand reasonably flexible, potentially resulting in cross-reactivity or easier degradation.

What we propose here is a novel and different approach, which involves the formation of polymerizable aptamer sequences prepared by introducing C-5 alkene-modified 2'deoxyuridine residues into the DNA strand, thus resulting in single to multiple covalent anchoring points between the aptamer and the polymer matrix. We produce MIP NPs imprinted for cocaine using a solid-phase imprinting and selection procedure ${ }^{[17]}$ either with a plain classical polymerization mixture (Plain MIP NPs) as well as several modified aptamer (AptaMIP NPs) and control sequences, demonstrating that the MIP NPs bearing the right aptamer sequence cross-linked with the MIP matrix through multiple anchoring points exhibit the best recognition performance for the template. Moreover, we demonstrate that the polymer matrix protects the degradation of DNA aptamer strand from nuclease.

The modified nucleoside monomer which has been used to develop this work is commercially available as phosphoramidite (Link, UK), or can be synthesized using a rapid microwavebased palladium-catalyzed modification of 5-iodo-2'-deoxyuridine ${ }^{[18]}$ (Figure 1a). 
(a)

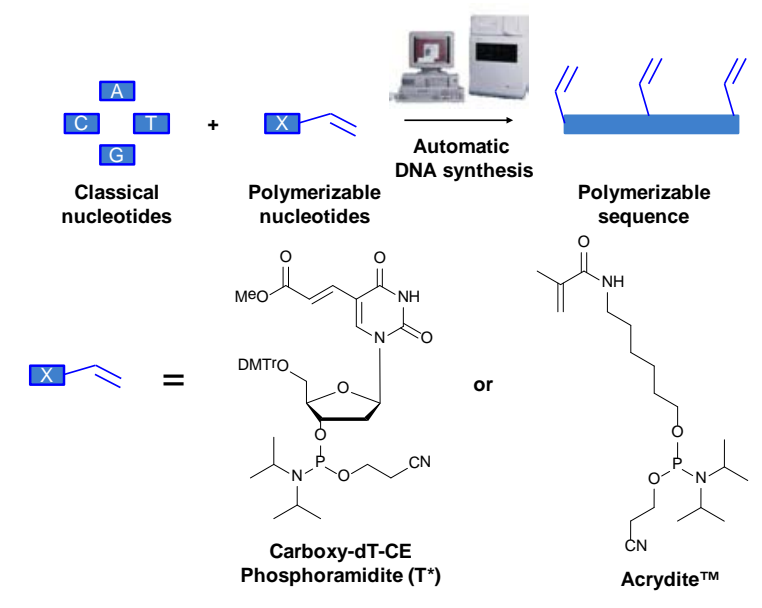

(b)

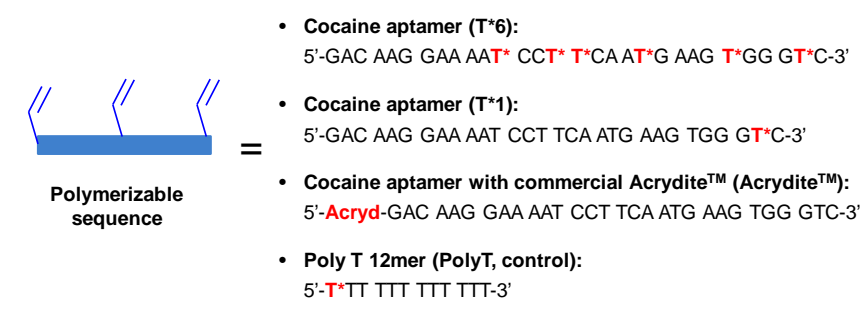

Figure 1. (a) Scheme showing the synthesis of polymerizable aptamer sequences using the modified Carboxy-dT-CE Phosphoramidite ( $\left.\mathrm{T}^{*}\right)$ or the Acrydite ${ }^{\mathrm{TM}}$. (b) List of polymerizable DNA sequences used in this work: cocaine aptamer with one $\left(\mathrm{T}^{*} 1\right)$ or six $\left(\mathrm{T}^{*} 6\right)$ polymerizable residues or with a flexible 5'-terminal modification (Acrydite ${ }^{\mathrm{TM}}$ ). Poly $\mathrm{T}$ 12mer sequence has been synthesized as a control (PolyT).

The aptamer sequence chosen (5'-GAC AAG GAA AAT CCT TCA ATG AAG TGG GTC$3^{\prime}$ ) was published for the template cocaine in $2001,{ }^{[19]}$ and since then it has been investigated and exploited in a number of studies. ${ }^{[20]}$ This sequence exhibits a total of six thymine residues, which could potentially be used for substitution with the modified polymerizable monomer (Figure 1b).

We decided to substitute one thymidine $\left(T^{*} 1\right)$ or all six thymidines $\left(T^{*} 6\right)$ to provide either single or multiple anchoring points with the polymer. During the oligonucleotide deprotection conditions, the ester group is transformed into an acrylamide. We have chosen this type of 


\section{WILEY-VCH}

modification because from our previous work other types of alkene or allyl modifications do not result in a sufficient incorporation level of the monomers into the polymer matrix (data not shown). On its own the nucleoside monomer has been already used in our group to perform the imprinting of 2'-deoxyadenosine nucleoside, and it has resulted in an improved rebinding and specific recognition performance in comparison with plain MIP NPs prepared using only the classical acrylamide monomers. ${ }^{[21]}$

For comparison purposes, we have also used a commercial Acrydite ${ }^{\mathrm{TM}}$ modified aptamer sequence (Figure 1b), as detailed in the work of Spivak et al. as well as other authors. ${ }^{[16,20 a \text {, }}$ ${ }^{20 f]}$ This modification is readily available from commercial suppliers but it might not represent the best option for an imprinting approach because it could confer too much flexibility to the aptamer sequence, thus resulting in a reduced recognition performance and poorer applicability for diagnostic purposes.

In addition, to confirm that a specific aptamer sequence is required in the polymer to obtain the recognition, a polymerizable Poly $\mathrm{T}$ 12mer containing one polymerizable monomer residue (PolyT) has also been synthesized.

The solid-phase MIP NPs synthesis and selection strategy used in this work involves the immobilization of the template onto glass beads as solid support, hence we exploited an EDC/NHS coupling procedure to immobilize a cocaine analogue and metabolite bearing a carboxylic group (benzoylecgonine, BEC) onto amino-derivatized glass beads (Figure 2). 


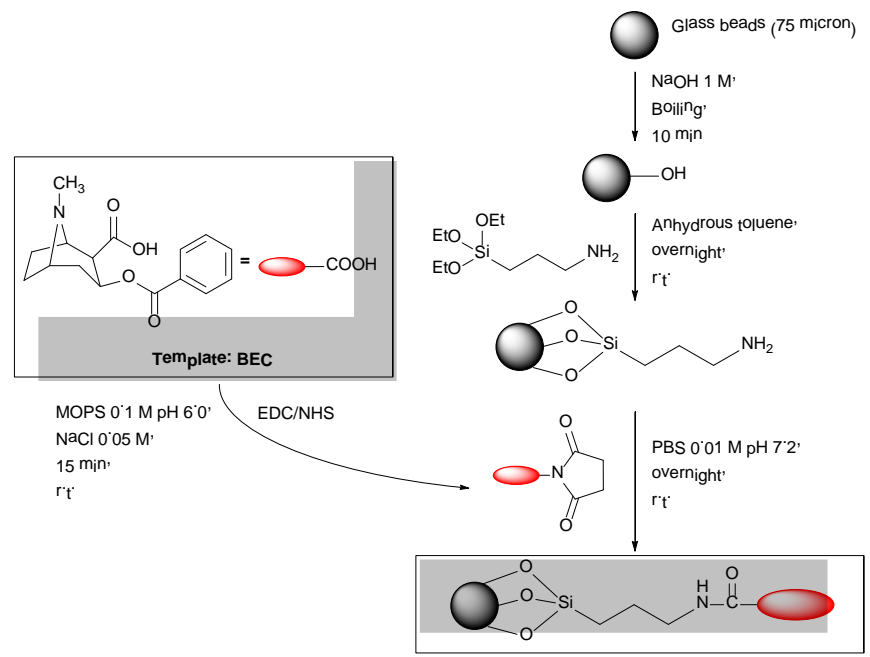

Figure 2. Schematic of the synthetic protocol for the immobilization of the cocaine analogue template (benzoylecognine, BEC) on the glass beads surface.

Specifically, the beads were previously activated in boiling $\mathrm{NaOH}$ solution to increase the number of reactive $\mathrm{OH}$ groups on their surface, which then facilitates the following step, resulting in a good coverage of the glass surface with silane containing primary amino groups (potentially up to $12 \mu \mathrm{mol}$ per gram of solid phase). ${ }^{[22]}$ After the silanization step, the activation of the carboxylic group of BEC using EDC/NHS allowed the immobilization of the template molecules forming an amide bond with the amino groups present on the glass beads. Using this strategy, the amount of BEC templates immobilized, determined spectrophotometrically (at $\lambda_{\max }=231 \mathrm{~nm}$ ) was $0.13 \mu \mathrm{mol} \mathrm{g}{ }^{-1}$ of glass beads.

After washing to remove the unbound BEC, the solid-phase synthesis of MIP NPs was then performed in the presence of template immobilized on glass beads. ${ }^{[17,23]}$ The principle behind this method is summarized in Figure 3. 


\section{WILEY-VCH}

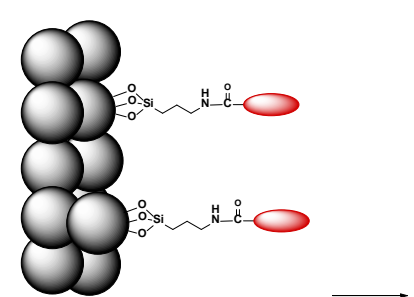

solid phase with ¡mmobilize d template $+$

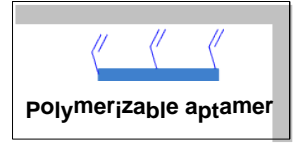

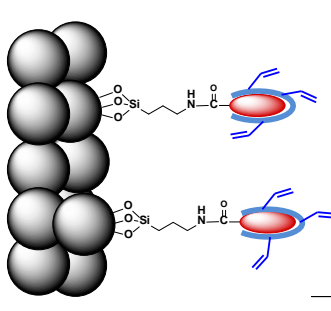

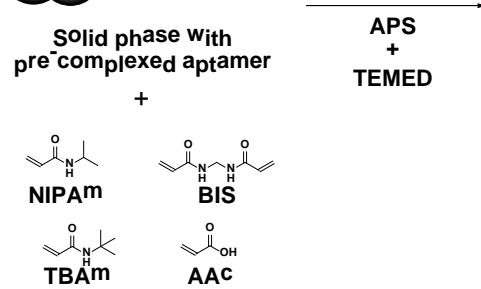

polymerization $m_{i} x_{t}$ ure
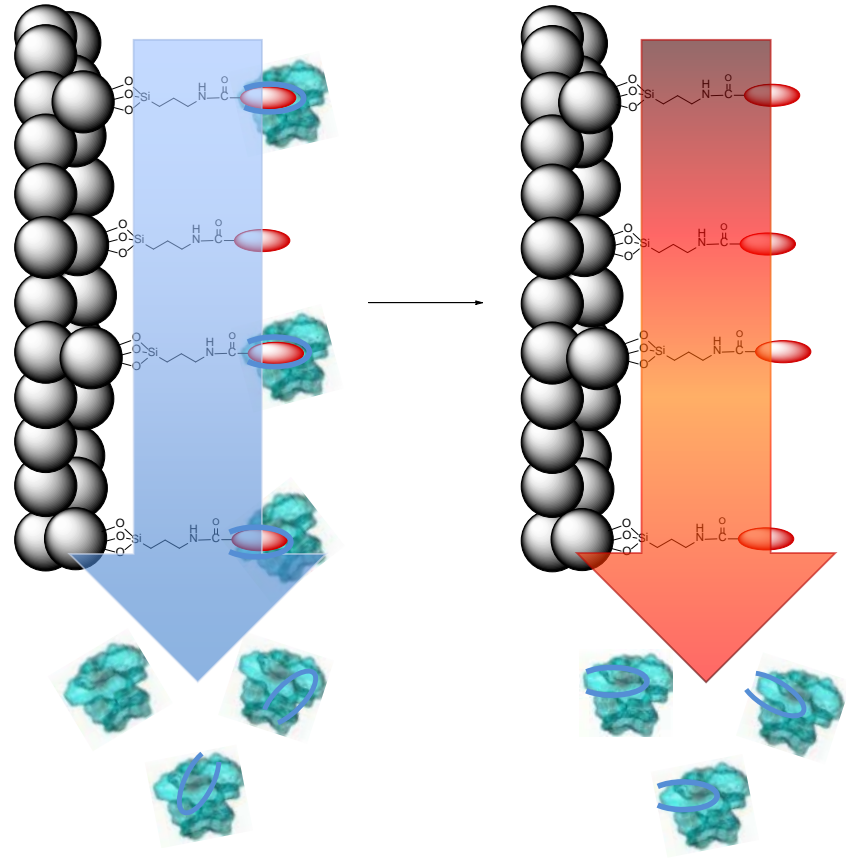

$60^{\circ} \mathrm{C}, \mathrm{H}_{2} \mathrm{O}$ : Elution of high"affinity MIP NPS

$20^{\circ} \mathrm{C}$ ' PBS: Elution of low־affinity NPS an shorter polymer fractions, monomers and

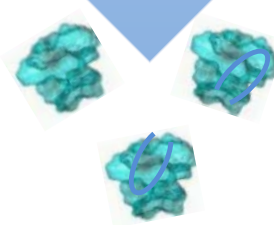

impurities

Figure 3. Schematic representation of the solid-phase synthesis and selection of AptaMIP NPs. The polymerizable sequence is incubated first with the solid phase bearing the cocaine analogue template (BEC). After this step the remaining classical monomers are added to the solid phase bearing at this point the immobilized template complexed with the aptamer DNA and the polymerization is initiated by the addition of APS and TEMED. In the case of Plain MIP NPs, the first incubation step is performed using simple PBS without any DNA aptamer. The low-affinity particles, as well as unreacted monomers, are washed at relatively low temperature using PBS (0.005 M, pH 7.4). The temperature is then increased and high-affinity MIP NPs are eluted from the solid phase using water. Scheme adapted from Poma et al. ${ }^{[17 \mathrm{a}]}$

First, the solid phase was suspended in the aptamer monomer solution (either $\mathrm{T}^{*} 1, \mathrm{~T}^{*} 6$, Acrydite $^{\mathrm{TM}}$ or PolyT) and incubated for $1 \mathrm{~h}$. This would allow correct folding of the aptamer sequences onto the immobilized template, thus producing a "pre-polymerization” complex which should then act as a polymerization center for the subsequent nanoparticle formation. We used a 2-fold excess of BEC in comparison to the molar amount of aptamer; this choice 


\section{WILEY-VCH}

was dictated by the limited amount of polymerizable aptamer material available. The concentration of the sequences used was slightly below the range of $K_{\mathrm{D}} \mathrm{s}$ measured for this type of aptamer (58 $\mu \mathrm{M}$, while the measured $K_{\mathrm{D}}$ S range from $80 \mu \mathrm{M}$ to $\left.200 \mu \mathrm{M}\right){ }^{\left[19,{ }^{20]}\right.}$ This transduced into a maximum theoretical amount of aptamer-BEC complexes ranging between 29 and $55 \mu \mathrm{M}$. In the case of Plain MIP NPs, the first incubation step is performed using simple PBS without any DNA aptamer. After the incubation, the remaining monomer solution composed of NIPAm, BIS, TBAm and AAc was poured in the reaction vessel and the polymerization process was then initiated in mild conditions by adding a redox-initiation system (APS and TEMED). The polymerization process used here has been extensively optimized and exploited by several groups for producing MIP NPs in aqueous media imprinted with all sorts of "delicate" structures, ranging from peptide sequences to whole proteins. ${ }^{[17,24]}$ This is one of the first examples in which this system has been exploited to imprint such a small structure like cocaine, which in this case has been chosen as a model template not only due to the importance of its detection in those cases of suspected drug abuse, but also because the aptamer sequence for its detection is very well characterized. ${ }^{[19,20 \mathrm{~d}]}$ As the reaction proceeds, polymer formed around the template will be retained due to its affinity and this allows for the subsequent washing/removal of low-affinity polymer and any unreacted monomer (Figure 3). In this case, in addition to the temperature we exploited also ionic strength to perform the selection procedure on the BEC-derivatized glass beads. The unreacted monomers and other low-affinity materials were washed out at $20{ }^{\circ} \mathrm{C}$ using PBS, while the high-affinity products were ultimately eluted from the template-derivatized glass beads by percolating double-distilled $\mathrm{H}_{2} \mathrm{O}$ at $60{ }^{\circ} \mathrm{C}$ (Figure 3). The increase in temperature and the reduction in ionic strength disrupt the interactions between the immobilized target and the high-affinity MIP NPs, thus assisting in their elution and collection. This procedure results in an average yield of $37 \pm 3 \%$ (w/w) of high-affinity product with respect to initial monomer mass (Table S2), without showing dependence on the type of polymerizable DNA sequence 


\section{WILEY-VCH}

used during the polymerization. As previously demonstrated, ${ }^{[17]}$ this solid-phase imprinting technique is extremely convenient for the production of high-affinity MIP NPs, offering several advantages in comparison with classical preparation procedures: ${ }^{[12 a]}$ i) formation of highly accessible binding sites (surface imprinting); ${ }^{[25]}$ ii) possible automation; ${ }^{[17]}$ iii) postderivatization or labelling of the nanoparticles for diagnostic, stabilization or immobilization purposes; ${ }^{[23,26]}$ iv) recyclability of templates, ${ }^{[17 b, 21,24 e]}$ which is a very important aspect in this case due to its regulated accessibility and elevated cost ( £450 for $100 \mathrm{mg})$. In addition, MIP NPs can be collected as a pure fraction, free from template and monomers, in a maximum time of $24 \mathrm{~h}$ per batch, as compared to days needed with methods which rely on dialysis. ${ }^{[24]}$ This time could potentially be reduced even more, but in this case we did not want to risk minimizing the incorporation of the DNA monomer inside the nanoparticles, hence we maintained the same polymerization times as optimized for the nucleoside monomer on its own. ${ }^{[21]}$

Under these conditions the incorporation level achieved for the aptamer sequences into the MIP NPs ranged from 55 to $75 \%(w / w)$ of the initial feed ratio (Table 1).

Table 1. Polymerizable DNA monomer incorporation levels into MIP NPs.

\begin{tabular}{lc}
\hline $\begin{array}{l}\text { Polymerizable DNA } \\
\text { monomer }\end{array}$ & $\begin{array}{c}\text { Incorporation level } \\
(\%, \mathrm{w} / \mathrm{w})\end{array}$ \\
\hline Acrydite $^{\mathrm{TM}}$ & $62.9 \%$ \\
$\mathrm{~T}^{\star} 1$ & $55.7 \%$ \\
$\mathrm{~T}{ }^{* 6}$ & $55.9 \%$ \\
PolyT & $74.3 \%$ \\
\hline
\end{tabular}

It is surprising that the number of the polymerizable moieties did not significantly affect the incorporation performance. The incorporation level was comparable whether the aptamer sequence was bearing a single $\mathrm{T}^{*}$ or six $\mathrm{T}^{*}$. The Acrydite ${ }^{\mathrm{TM}}$ modification reached a slightly higher incorporation level, probably due to the higher availability and flexibility of the 


\section{WILEY-VCH}

double-bond moiety which is attached to the DNA aptamer through a spacing sequence. The PolyT sequence reached an even higher incorporation level, possibly due to the shorter length in comparison with the other DNA sequences tested (12 vs 30 nucleotides), which might result in an easier accessibility as well as mobility of the double-bond moiety during the polymerization process. Changing the monomer mixture or the polymerization conditions (e.g., time or monomer) might result in an increased incorporation level, ${ }^{[27]}$ but in this instance we did not want to use excessively harsh polymerization conditions because, as we mentioned initially, another important aspect of this work is to demonstrate the stability of the DNA aptamer sequence inside the nanoparticle matrix. This stability should derive entirely from its covalent connections established on the newly-introduced double-bond moieties rather than on secondary covalent connections established because of "thymine-dimer" structures formed following the usage of more drastic initiation systems such as UV. ${ }^{[27]}$ In addition, we wanted to make sure that the polymerization would take place in conditions mild enough to guarantee the correct folding of the DNA sequence around the template, as well as ensuring the obtainment of a "true" nanosystem rather than a sub-micro or microsystem. ${ }^{\text {[24] }}$

The hybrid polymerization strategy here used has resulted in the formation of NPs of about 10-30 nm in diameter, regular in size and spheroidal in shape (see Figure 4 for a typical TEM of Plain, PolyT and AptaMIP NPs T*1, T*6 and Acrydite ${ }^{\mathrm{TM}}$ ). 


\section{WILEY-VCH}

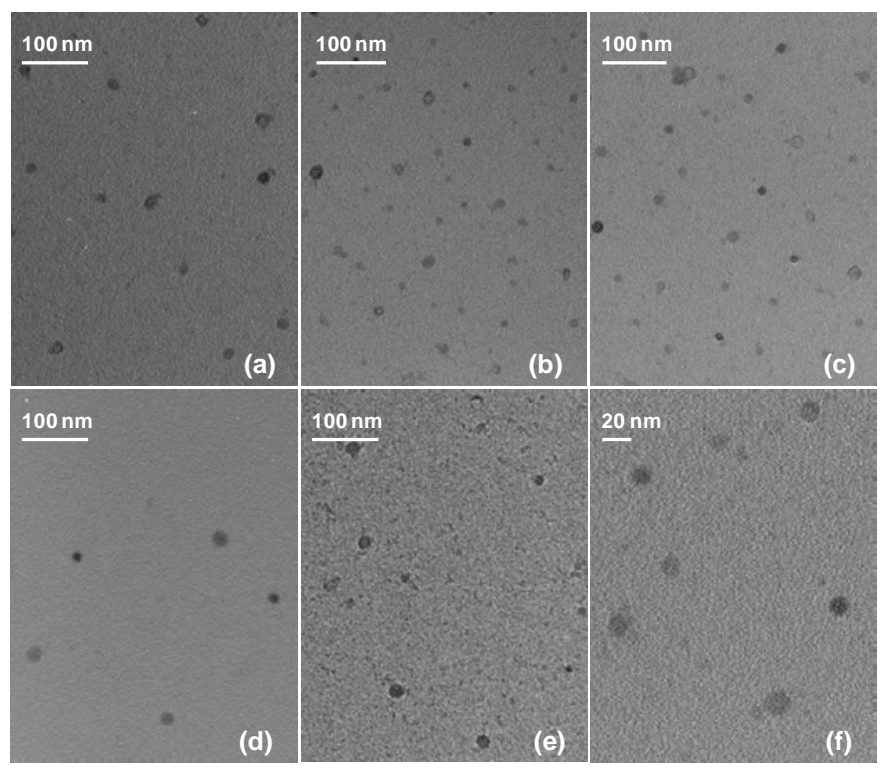

Figure 4. A typical TEM image of: (a) AptaMIP NPs Acrydite ${ }^{\mathrm{TM}}$; (b) AptaMIP NPs T*1; (c, f) AptaMIP NPs T*6; (d) PolyT MIP NPs; (e) Plain MIP NPs. All images have been taken at $30000 \times$ magnification, except (f) which is an inset of (c) at $60000 \times$ magnification to detail the spheroidal shape of the MIP NPs.

The presence or absence of the aptamer monomer in the preparation did not affect the size. In addition neither sedimentation nor visible aggregation phenomena were observed during storage (even after four months at $4{ }^{\circ} \mathrm{C}$ ).

Despite the similar levels of DNA monomer incorporation observed for the three aptamer MIP NPs, a definitive difference in rebinding performance was exhibited when the NPs were tested using microgravimetric analysis performed on a Stanford Research Systems QCM200 5 $\mathrm{MHz}$ quartz crystal microbalance. ${ }^{[28]}$ BEC template was immobilized onto the gold crystal surface using the same immobilization conditions exploited on the solid phase used during imprinting. This will ensure that the template is oriented in the same way as during production of the MIP NPs. Several concentrations (from of 0.125 to $2 \mu \mathrm{g} \mathrm{mL} \mathrm{L}^{-1}$ ) of the high-affinity fraction of AptaMIP NPs ( $\mathrm{T}^{*} 1, \mathrm{~T}^{*} 6$, Acrydite ${ }^{\mathrm{TM}}$ ), PolyT MIP NPs or Plain or Control NPs (i.e., imprinted for dC) were sequentially flowed (from the lowest to the highest 


\section{WILEY-VCH}

concentration) on the chip bearing BEC, and their binding behavior was recorded. A schematic representation of the QCM assay is represented in Figure 5, while the results of the study are summarized in Figure 6.

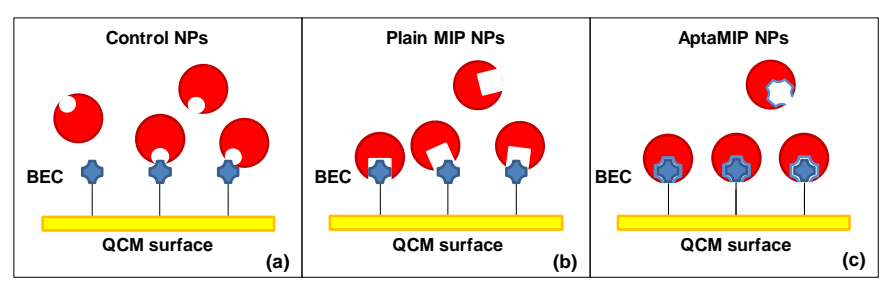

Figure 5. Schematic representation of the binding to immobilized BEC of: (a) control NPs (MIP NPs imprinted for dC); (b) Plain MIP NPs; (c) AptaMIP NPs. The introduction of the aptamer monomer with multiple anchoring points improves the recognition performance towards the imprinted template.

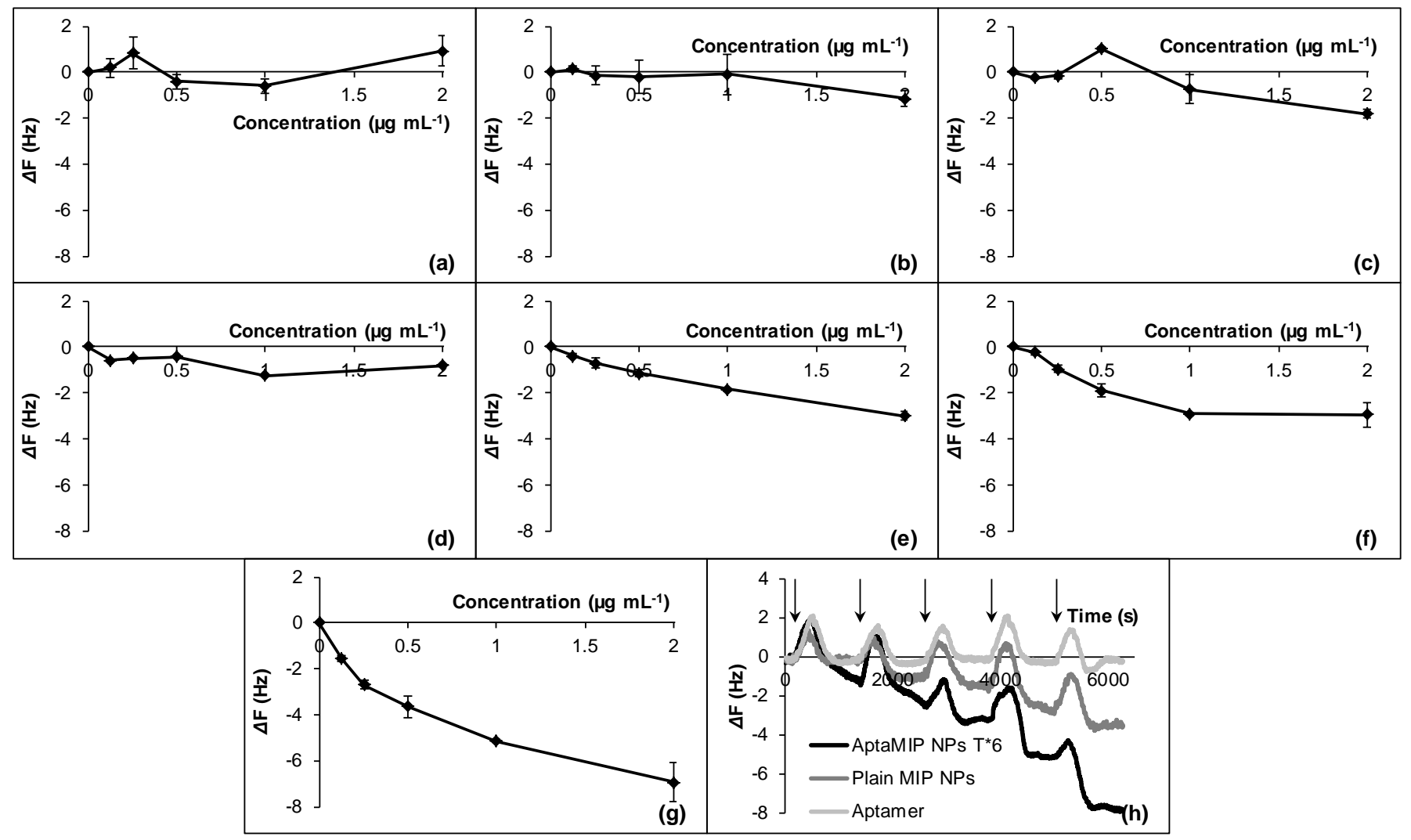

Figure 6. Rebinding to BEC surface of: (a) aptamer (on its own); (b) Control MIP NPs (imprinted for dC); (c) PolyT MIP NPs; (d) AptaMIP NPs T*1; (e) AptaMIP NPs Acrydite ${ }^{\mathrm{TM}}$; 


\section{WILEY-VCH}

(f) Plain MIP NPs; (g) AptaMIP NPs T*6. A typical outcome of a QCM experiment of Aptamer, AptaMIP NPs T*6 and Plain MIP NPs on a BEC-derivatized surface is depicted in (h); absorption to the template is characterized by a drop in frequency on exposure to the sample solution. Arrows indicate the points of injection. Concentration of all samples ranged from 0.125 to $2 \mu \mathrm{g} \mathrm{mL} \mathrm{m}^{-1}$. QCM measurements were performed in PBS (0.003 M, pH 7.4) at $20{ }^{\circ} \mathrm{C}$. Of note are the "peaks” after injection of the samples (Figure $7 \mathrm{~h}$ ). These are due to the relative changes in pressure during the injection process. All points are measured after system has stabilized in buffer after injection. Data for 6a, 6b, 6c, 6d, 6e, 6f and $6 \mathrm{~g}$ are differences from original starting frequency and were calculated from these stabilized periods. Error bars represent $\pm 1 \mathrm{SD}(n=3)$.

The sensorgrams presented here show the ability of Plain MIP NPs and AptaMIP NPs T*6 and Acrydite ${ }^{\mathrm{TM}}$ (Figure 6e, 6f, 6g, 6h) to recognize and bind their target specifically.

The aptamer on its own did not exhibit any relevant binding when its rebinding performance was assessed using the microgravimetric platform (Figure 6a and 6h). The lack of observed binding by the aptamer is probably due to the sensitivity of the QCM system, with the aptamer itself not possessing enough mass to change the frequency, given that there will be limited recognition sites on the surface. The greater bulk of the MIP NP allows the system to register binding, by mass. Given the incorporation data (Table 1), the calculated sizing by TEM (Figure 4), and the identical solid-phase synthesis approach for all the MIP NPs, the mass and density of the MIP NPs is likely to be comparable and QCM results among them reflect differences in binding.

The control NPs imprinted for $\mathrm{dC}$ did not show any binding (Figure 6b), demonstrating the effectiveness of the template specificity of the imprinting procedure.

No binding was exhibited when PolyT MIP NPs were analyzed (Figure 6c). Indeed the presence of the right DNA sequence suitable to achieve the correct folding around the 


\section{WILEY-VCH}

template molecule is needed to exhibit a certain rebinding performance. Interestingly, AptaMIP NPs bearing only one polymerizable residue $\left(\mathrm{T}^{*} 1\right)$ did not exhibit any significant binding (Figure 6d). We hypothesize that a single polymerizable residue positioned so close to the aptamer sequence may lock the aptamer into an inappropriate conformation. This does not seem to be the case for the Acrydite ${ }^{\mathrm{TM}}$ modification (Figure 6e), which also bears a single double-bond but in position 5' rather than 3' and is connected to the aptamer sequence through a spacer. In this case the connection through the spacer sequence might ensure a better folding of the aptamer onto the template, and subsequent improved imprinting procedure helped by the polymer matrix. Indeed, the Plain MIP NPs imprinted for BEC without using any polymerizable DNA sequence also exhibited a similar rebinding performance (Figure $6 \mathrm{f}$ and $6 \mathrm{~h}$ ). In both cases (Plain MIP NPs and AptaMIP NPs Acrydite ${ }^{\mathrm{TM}}$ ) there was a total frequency drop of about $3 \mathrm{~Hz}$.

The explanation for the binding behavior of AptaMIP NPs $\mathrm{T}^{*} 1$ and Acrydite ${ }^{\mathrm{TM}}$ may be understood in light of the aptamer structure described by Cekan et al. ${ }^{[29]}$ The aptamer folds into three helixes, one of which (containing both 5' and 3' ends) forms upon binding to cocaine. It is possible that the higher flexibility conferred to the aptamer by the spacer included in the Acrydite ${ }^{\mathrm{TM}}$ modification allows the third helix to form more easily upon binding to cocaine, while in the case of $\mathrm{T}^{*} 1$ a modification so close to the base in the absence of further anchoring points along the DNA sequence might hinder duplex formation or template binding. In any case, there is a clear influence of both the nature and site of polymerizable modification on the effectiveness of AptaMIP NPs synthesis with multiple modifications showing a clear advantage.

Indeed, AptaMIP NPs $\mathrm{T}^{*} 6$ exhibited a drop in frequency of almost $7 \mathrm{~Hz}$ when tested by QCM onto a BEC-derivatised surface (Figure $6 \mathrm{~g}$ and $6 \mathrm{~h}$ ). A calculated apparent dissociation constant $\left(K_{\mathrm{D}}\right)$ of $4 \mathrm{nM}$ was obtained, whilst in the case of Plain and AptaMIP NPs Acrydite ${ }^{\mathrm{TM}}$, the $K_{\mathrm{D}}$ values were respectively $13.7 \mathrm{nM}$ and $26.4 \mathrm{nM}$. This confirms that the calculated 


\section{WILEY-VCH}

affinity for the target for the particles was higher than the aptamer sequence on its own, given by the literature as between $80 \mu \mathrm{M}$ to $200 \mu \mathrm{M} \cdot{ }^{[19,20]}$ These results suggest that the presence of multiple covalent anchoring points between the aptamer and the imprinted matrix is beneficial to the interaction between aptamer and target.

It would be interesting to test whether larger templates like proteins can be exploited to produce AptaMIPs using the method described in this work. ${ }^{[16]}$ Likewise, performing a UV polymerization process might result in a higher incorporation as well as rigidity of the polymerizable aptamer monomer and associated increase in recognition. ${ }^{[27]}$ Both these aspects are currently under investigation.

To assess the stability of the DNA aptamer inside the AptaMIP NPs, the T*6 and Acrydite ${ }^{\mathrm{TM}}$ AptaMIP NPs, which had exhibited the best rebinding performance, were incubated with nuclease $\mathrm{P}_{1}$, which is widely used for endonucleolytic digestion of single-stranded DNA. ${ }^{[30]}$ After the incubation period, centrifugal ultrafiltration allowed to separate the enzyme and the AptaMIP NPs from the digested DNA, which was then determined spectrophotometrically (at $\lambda_{\max }=260 \mathrm{~nm}$ ) by analyzing the washings. No DNA digestion was observed from the T*6 or Acrydite $^{\mathrm{TM}}$ AptaMIP NPs, while under the same conditions, nearly $100 \%$ of free DNA is digested to single nucleotides. Thus the polymer matrix is suitable to protect the aptamer from enzymatic digestion. More studies are in progress to assess the stability of the AptaMIP NPs in other conditions.

In conclusion, this paper describes the first example of solid-phase synthesis of aptamerimprinted polymer hybrid nanomaterials capable of molecular recognition. Specifically we have successfully produced MIP NPs for BEC (cocaine analogue) by exploiting a modified polymerizable aptamer, containing either a commercial modification at the 5' end (AptaMIP NPs Acrydite ${ }^{\mathrm{TM}}$ ) or single or multiple C-5 alkene-modified uridine nucleotides (AptaMIP NPs $\mathrm{T}^{* 1}$ and $\left.\mathrm{T}^{*} 6\right)$. Nanoparticles were obtained using a solid-phase imprinting polymerization 


\section{WILEY-VCH}

strategy in which template-derivatized glass beads double as an affinity matrix for production as well as selection and purification of synthesized imprinted nanoparticles.

The nanomaterials obtained are spheroidal in shape, with a size comparable to natural antibodies. ${ }^{[31]}$ The solid-phase imprinting strategy guarantees ease of accessibility of the binding sites, resulting de facto in a surface-imprinting procedure.

Plain and AptaMIP NPs recognized BEC specifically when assessed by QCM, and the best rebinding performance was obtained, as expected, when multiple anchoring points were introduced into the aptamer sequence, in the case of AptaMIP NPs T*6. Surprisingly, AptaMIP NPs $\mathrm{T}^{* 1}$ did not exhibit any significant rebinding and further investigations are being undertaken to better understand the parameters defining optimal incorporation of polymerizable modifications in AptaMIP NPs, including the optimum site, number, flexibility and type of modification. Future work is also planned to assess the effect of different amounts of aptamer DNA included in the preparation on the size, affinity and specificity of the hybrid AptaMIP NPs. The use of a more sensitive detection system such as Surface Plasmon Resonance (e.g. Biacore ${ }^{\circledR}$ ) will be investigated for future analysis. In the early days of this field it is likely that an empirical process of testing several modification patterns will be necessary for each new aptamer sequence, to find AptaMIPs with optimal binding properties. Importantly, AptaMIP NPs are resistant to digestion by nuclease $\mathrm{P}_{1}$, thus confirming that the polymer matrix acts as a shield for the incorporated aptamer sequence.

We believe that this approach might be further explored to potentially produce hybrid MIPDNA systems with improved recognition performance also for larger templates, or even with more complex architectures which are more suitable for diagnostic applications, such as coreshell systems. ${ }^{[23,26]}$ The gentle polymerization conditions here used should guarantee that the aptamer monomers would retain their stability, potentially leading to hybrid AptaMIP NPs suitable for in vitro or even in vivo applications such as drug development and delivery 


\section{WILEY-VCH}

systems. $^{[12 b, 32]}$ We are actively exploring the potential of AptaMIP NPs within these applications.

\section{Experimental Section}

Materials: $\quad N$-isopropylacrylamide $\quad$ (NIPAm), $\quad N, N, N^{\prime}, N^{\prime}$-tetra-methylethylenediamine (TEMED), ammonium persulphate (APS), acrylic acid (AAc), $N, N^{\prime}$-methylenebisacrylamide (BIS), $\quad N$-tert-butylacrylamide (TBAm), 3-aminopropyltriethyloxy-silane (APTES), benzoylecgonine tetrahydrate (BEC), cysteamine, glass beads, SPE cartridges and frits, toluene, methanol, acetone, zinc chloride and nuclease $\mathrm{P}_{1}$ (EC: 3.1.30.1) were purchased from Sigma-Aldrich (UK). Sodium hydroxide, sulfuric acid, zinc chloride, sodium acetate, acetic acid, 1-ethyl-3-(3-dimethylaminopropyl)carbodiimide (EDC), $\mathrm{N}$-hydroxysuccinimide (NHS), 3-(N-morpholino)propanesulfonic acid (MOPS), tris(hydroxymethyl)aminomethane (TRIS) and phosphate buffered saline (PBS) were purchased from Fisher Scientific (UK). Ethanol and hydrogen peroxide were purchased from VWR (UK). Cocaine aptamer sequences bearing the Acrydite ${ }^{\mathrm{TM}}$ modification were purchased from Integrated DNA Technologies, Inc (USA). Carboxy-dT-CE Phosphoramidite and 2'-deoxycytidine (dC) were purchased from Link (UK). Double-distilled water (Millipore) was used for analysis. All chemicals and solvents were analytical or HPLC grade and were used without further purification.

Synthesis of polymerizable oligomer and aptamer sequences (Aptamer $T^{*} 1$, Aptamer $T^{*} 6$ and PolyT): Oligonucleotides were synthesized under standard conditions at $1 \mu \mathrm{mol}$ scale on an Applied Biosystems 394 oligonucleotide synthesizer. The oligomers were deprotected and released from the support by treatment with concentrated aqueous $\mathrm{NH}_{3}$ at $55{ }^{\circ} \mathrm{C}$ for $16 \mathrm{~h}$. The solutions were concentrated to dryness, resuspended in water and desalted using NAP-10 columns (GE Healthcare). Oligonucleotide masses were verified using a Bruker micrOTOF LCMS system. 


\section{WILEY-VCH}

Preparation of BEC-derivatized glass beads as affinity media: Glass beads (125 g, $75 \mu \mathrm{m}$ diameter, Supelco) were activated by boiling in $\mathrm{NaOH}(1 \mathrm{M})$ for $10 \mathrm{~min}$, then washed thoroughly with double-distilled water at $60{ }^{\circ} \mathrm{C}$, acetone and finally dried at $80{ }^{\circ} \mathrm{C}$. They were then incubated in a solution of APTES (2\%, v/v) in anhydrous toluene overnight at room temperature, then washed with acetone and dried under vacuum. The BEC template (10 mg) was activated in $1 \mathrm{~mL}$ MOPS buffer (0.1 M, NaCl $0.05 \mathrm{M}$, pH 6.0) by adding $5 \mu \mathrm{L}$ EDC and $6 \mathrm{mg}$ NHS for $15 \mathrm{~min}$, then this solution was diluted with PBS (0.01 M, pH 7.2) to $30 \mathrm{~mL}$ and used to incubate $45 \mathrm{~g}$ of APTES-derivatized glass beads overnight at room temperature (0.67 $\mathrm{mL}$ solution/g glass beads). The derivatized beads were washed thoroughly with doubledistilled water and dried under vacuum. After this step the glass beads were used straight away for the synthesis of the MIP NPs without further storage. The amount of template immobilized was determined spectrophotometrically (at $\lambda_{\max }=231 \mathrm{~nm}$ ) by analyzing the amount of BEC unbound to the glass beads and found in the washings collected from the immobilization step.

Solid-phase synthesis of BEC-imprinted MIP and AptaMIP NPs: a solution (0.29 $\mu$ moles in $2.5 \mathrm{~mL}$ ) of each polymerizable DNA sequence in PBS (0.005 M, pH 7.4) was degassed by purging Ar for $10 \mathrm{~min}$ and then incubated for $1 \mathrm{~h}$ at room temperature in a $14 \mathrm{~mL}$ glass vial closed using a Teflon screw-cap and containing $5 \mathrm{~g}$ of template BEC-derivatized glass beads (0.5 mL solution/g glass beads), for a total of six polymerization vials (Plain MIP NPs, AptaMIP NPs Acrydite ${ }^{\mathrm{TM}}$, AptaMIP NPs T*1, AptaMIP NPs T*6, PolyT MIP NPs). This corresponds to a ratio of 2.24 molecules of BEC per each molecule of aptamer sequence. In the case of Plain MIP NPs, 2.5 mL of PBS were added to maintain the incubation conditions similar to the other samples. Prior to the addition of the aptamer solution, the vials containing the solid phase were degassed under vacuum and the air inside the vials then replaced with $\mathrm{Ar}$ (3 times). In the meantime the following monomers were dissolved in PBS (0.005 M, pH 7.4, 50 mL): NIPAm (39 mg, 0.35 mmol, 53\%), BIS (2 mg, 0.01 mmol, 2\%), TBAm (33 mg, 0.26 


\section{WILEY-VCH}

mmol, 40\%) and AAc (2.2 $\mu \mathrm{L}, 0.03 \mathrm{mmol}, 5 \%)$. TBAm was previously dissolved in EtOH (1 $\mathrm{mL}$ ) and then added to the aqueous solution. The total monomer concentration was $13 \mathrm{mM}$ at this stage. The solution was degassed under vacuum and sonication for $10 \mathrm{~min}$, and then purged with Ar for $30 \mathrm{~min}$. After this time, aliquots of $2.5 \mathrm{~mL}$ of solution were transferred in the vials previously incubated with the polymerizable DNA, thus reaching a total volume of 5 $\mathrm{mL}$ and a final monomer concentration of $6.5 \mathrm{mM}$. The polymerization was started by adding an APS aqueous solution (50 $\left.\mu \mathrm{L}, 60 \mathrm{mg} \mathrm{mL}^{-1}\right)$ and TEMED $(1.5 \mu \mathrm{L})$. The polymerization was then carried out at $20^{\circ} \mathrm{C}$ for $20 \mathrm{~h}$. After the polymerization, the contents of the vials were transferred into empty SPE cartridges fitted with a polyethylene frit (20 $\mu \mathrm{m}$ porosity) in order to perform the temperature-based affinity separation of MIP NPs. The temperature of PBS and the SPE cartridges was kept at $20{ }^{\circ} \mathrm{C}$ (same as the polymerization step). Washing was performed with $3 \times 5 \mathrm{~mL}$ of PBS (0.005 M, pH 7.4), applying manual pressure with a syringe if needed. This was done in order to remove non-polymerized monomers and low-affinity MIP NPs. The effectiveness of the washing was verified by measuring the UV absorbance of washing aliquots, in order to ensure complete monomer removal as well as to quantify the incorporation of polymerizable DNA into the polymer matrix (by difference of the absorbance measured at $\lambda=260 \mathrm{~nm}$, considering that 1 O.D. unit at that wavelength corresponds to 38.8 $\mu g$ of PolyT, $29.6 \mu \mathrm{g}$ of $\mathrm{T}^{*} 1,30.5 \mu \mathrm{g}$ of $\mathrm{T}^{*} 6$ or $31.2 \mu \mathrm{g}$ of Acrydite $\left.{ }^{\mathrm{TM}}\right)$. Afterwards the SPE cartridges containing the solid phase with high-affinity MIP NPs attached were heated up to $60{ }^{\circ} \mathrm{C}$ and eluted with $5 \times 5 \mathrm{~mL} \mathrm{H}_{2} \mathrm{O}$ at $60{ }^{\circ} \mathrm{C}$. Control NPs have been prepared in the same way but using $\mathrm{dC}$ as template. The concentration of the nanoparticles fractions has been evaluated by evaporation.

Transmission Electron Microscopy (TEM) analysis: TEM images of MIP NPs were taken using a JEOL JEM 1400, 120kV high contrast TEM equipped with an AMT XR60 midmount digital camera (11 megapixels). Samples for the analysis have been prepared by depositing a drop of the MIP NPs solution, previously filtered through a $0.45 \mu \mathrm{m}$ PTFE 


\section{WILEY-VCH}

syringe filter, on a carbon-coated TEM copper grid (300 mesh, from Agar Scientific, UK), blotting away the excess and leaving them to dry overnight at room temperature.

Dynamic Light Scattering (DLS) analysis: the samples for DLS were prepared in deionized $\mathrm{H}_{2} \mathrm{O}$, sonicated for $5 \mathrm{~min}$, then filtered through $0.45 \mu \mathrm{m}$ PTFE syringe filters and analyzed in a Quartz SUPRASIL $1.5 \times 1.5 \mathrm{~mm}$ cuvette at $25{ }^{\circ} \mathrm{C}$ by using a Malvern Viscotek DLS (Malvern Instruments Ltd.) equipped with OMNISIZE 3.0 software.

Treatment of Quartz Crystal Microbalance (QCM) crystals and surface immobilization of templates: QCM crystals (5 MHz Cr/Au, polished, Testbourne Ltd., UK) were cleaned by immersion in Piranha solution $\left(\mathrm{H}_{2} \mathrm{SO}_{4} / \mathrm{H}_{2} \mathrm{O}_{2}, 3 / 1\right.$, v/v) for 5 min. Caution! This mixture is highly corrosive, hence extreme care is required during this process. Then they were thoroughly rinsed with double-distilled water and left in $\mathrm{MeOH}$ overnight. The immobilization of the templates has been performed by incubating the crystals in a solution of cysteamine $\left(0.2 \mathrm{mg} \mathrm{mL}^{-1}\right)$ in EtOH at $4{ }^{\circ} \mathrm{C}$ for $24 \mathrm{~h}$, after which they have been washed with EtOH and incubated for $24 \mathrm{~h}$ at room temperature in a $0.5 \mathrm{mg} \mathrm{mL}^{-1}$ solution of BEC template in PBS (0.01 M, pH 7.2), previously activated in MOPS buffer (0.1 M, NaCl $0.05 \mathrm{M}, \mathrm{pH}$ 6.0) using EDC and NHS for 15 min (same activation as for the immobilization onto the glass beads). Once the immobilization was completed, the crystals were washed thoroughly with double-distilled water before being mounted in the QCM flowcell. Successful immobilization of the templates was confirmed through sessile water contact angle measurements performed on a Theta Optical Tensiometer (Biolin Scientific, UK).

QCM microgravimetric analysis of aptamer and BEC-imprinted Plain and AptaMIP NPs: Plain MIP NPs, PolyT MIP NPs, Control NPs (imprinted for dC), plain aptamer sequence and AptaMIP NPs ( $\mathrm{T}^{*} 1, \mathrm{~T}^{*} 6$ and Acrydite $\left.{ }^{\mathrm{TM}}\right)$ adsorption to BEC template was monitored using a

QCM200 5 MHz quartz crystal microbalance (Stanford Research Systems, UK). ${ }^{[28]}$ The modified QCM chips were maintained hydrated during mounting in the QCM flowcell. MIP NPs solutions and running buffer were introduced using an Instech P720 peristaltic pump 


\section{WILEY-VCH}

equipped with 0.020 ” ID tubing (Linton Instrumentation, UK) and flowing at $0.1 \mu \mathrm{L} \mathrm{min}^{-1}$. The QCM chip bearing the template was first stabilized in running buffer (PBS $0.003 \mathrm{M}, \mathrm{pH}$ 7.4) at $20^{\circ} \mathrm{C}$ until the system reached a stable baseline. Affinity analysis was carried out by sequentially flowing each MIP NPs solution for $5 \min (500 \mu \mathrm{L})$ and analyzing the sensor response for $15 \mathrm{~min}$. This process was repeated over the concentration range of 0.125-2 $\mu \mathrm{g}$ $\mathrm{mL}^{-1}$. The apparent $K_{\mathrm{D}} \mathrm{S}$ were calculated by nonlinearly fitting the binding data to a Langmuir binding isotherm model using GraphPad Prism 6.0 (GraphPad Software, US) under an assumption that all particles in solution are spheres of defined density. ${ }^{[24]}$

Nuclease $P_{1}$ DNA digestion test of AptaMIP NPs $T^{*} 6$ and Acrydite ${ }^{\mathrm{TM}}$ : the procedure was adapted from Li et al. ${ }^{[30]}$ An equivalent of AptaMIP NPs T*6 or Acrydite ${ }^{\mathrm{TM}}$ containing 0.2 mg DNA in $200 \mu \mathrm{L} \mathrm{H} \mathrm{H}_{2} \mathrm{O}$ in a $2 \mathrm{~mL}$ plastic tube is treated with $40 \mu \mathrm{L} \mathrm{ZnCl}_{2}(5 \mathrm{mM}), 60 \mu \mathrm{L}$ sodium acetate buffer (50 mM, pH 5.5) and $\mathrm{pH} 5.5$, and $0.5 \mathrm{U}(2 \mu \mathrm{L})$ nuclease $\mathrm{P}_{1}$ previously dissolved in TRIS buffer ( $30 \mathrm{mM}, \mathrm{pH}$ 7.5), giving a total volume of $302 \mu \mathrm{L}$ with $1.66 \mathrm{U} \mathrm{mL}^{-1}$ nuclease $\mathrm{P}_{1}, 0.66 \mathrm{mM} \mathrm{ZnCl}_{2}$, and $9.93 \mathrm{mM}$ sodium acetate. The tube is capped and kept for 2 h at $37{ }^{\circ} \mathrm{C}$ before centrifugal ultrafiltration (Amicon $0.5 \mathrm{~mL}$ centrifugal tubes 3k MWCO; Fisher Scientific, UK). Two washes of $250 \mu \mathrm{L} \mathrm{H}_{2} \mathrm{O}$ each of have been performed, to obtain a total volume of $750-800 \mu \mathrm{L}$. The amount of digested DNA was determined spectrophotometrically (at $\lambda_{\max }=260 \mathrm{~nm}$ ) by analyzing the washings collected from the ultrafiltration step.

\section{Supporting Information}

Supporting Information (UV calibration curve for the BEC template; sessile water contact angle measurements for the immobilization of BEC onto QCM crystals; yield and DLS data for Plain and AptaMIP NPs) is available from the Wiley Online Library or from the author.

\section{Acknowledgements}

The authors would like to acknowledge the financial support of The Open University and of Engineering and Physical Sciences Research Council (EPSRC) for the award of a First Grant (EP/K015095/1). AP would like to acknowledge Dr. Yao Xu and Dr. Sarah Allman for the 


\section{WILEY-VCH}

useful discussion on the synthesis of the polymerizable nucleoside and aptamer, and Mrs. Heather Davies for the help with the TEM imaging. The authors also acknowledge Prof. Cameron Alexander for the help with the DLS measurements.

Received: ((will be filled in by the editorial staff))

Revised: ((will be filled in by the editorial staff)) Published online: ((will be filled in by the editorial staff))

[1] a) A. Beck, T. Wurch, C. Bailly, N. Corvaia, Nat. Rev. Immunol. 2010, 10, 345; b) O. Leavy, Nat. Rev. Immunol. 2010, 10, 297.

[2] a) M. Johnson, Mater. Methods 2012, 2, 120; b) M. Mueller, M. T. Loh, D. Y. Tee, Y. Yang, A. Jungbauer, Appl. Biochem. Biotechnol. 2013, 169, 1431.

[3] a) V. J. B. Ruigrok, M. Levisson, M. H. M. Eppink, H. Smidt, J. van der Oost, Biochem. J. 2011, 436, 1; b) Y. Song, W. Wei, X. Qu, Adv. Mater. 2011, 23, 4215.

[4] M. Zhao, T. Wu, X. Xiao, Y. Liu, X. Su, Anal. Bioanal. Chem. 2013, 405, 5679.

[5] S. Banta, K. Dooley, O. Shur, Annu. Rev. Biomed. Eng. 2013, 15, 93.

[6] a) T. Smuc, I. Y. Ahn, H. Ulrich, J. Pharm. Biomed. Anal. 2013, 81-82, 210; b) W. Tan, H. Wang, Y. Chen, X. Zhang, H. Zhu, C. Yang, R. Yang, C. Liu, Trends Biotechnol. 2011, 29, 634.

[7] S. D. Jayasena, Clin. Chem. 1999, 45, 1628.

[8] R. E. Wang, H. Wu, Y. Niu, J. Cai, Curr. Med. Chem. 2011, 18, 4126.

[9] P. Dua, S. Kim, D. K. Lee, Recent Pat. DNA Gene Seq. 2008, 2, 172.

[10] a) C. Alexander, H. S. Andersson, L. I. Andersson, R. J. Ansell, N. Kirsch, I. A. Nicholls, J. O'Mahony, M. J. Whitcombe, J. Mol. Recognit. 2006, 19, 106; b) M. J. Whitcombe, N. Kirsch, I. A. Nicholls, J. Mol. Recognit. 2014, 27, 297; c) B. Sellergren, Nat. Chem. 2010, 2, 7; d) H. Asanuma, T. Hishiya, M. Komiyama, Adv. Mater. 2000, 12, 1019.

[11] a) M. J. Whitcombe, MIP Database, http://www.mipdatabase.com/, accessed: 17/08/2014; b) S. Subrahmanyam, S. A. Piletsky, E. V. Piletska, B. Chen, R. Day, A. P. F. 


\section{WILEY-VCH}

Turner, Adv. Mater. 2000, 12, 722; c) B. Sellergren, B. Rückert, A. J. Hall, Adv. Mater. 2002, 14, 1204; d) R. Schirhagl, P. A. Lieberzeit, F. L. Dickert, Adv. Mater. 2010, 22, 2078; e) M. A. Brenckle, H. Tao, S. Kim, M. Paquette, D. L. Kaplan, F. G. Omenetto, Adv. Mater. 2013, 25, 2409.

[12] a) A. Poma, A. P. F. Turner, S. A. Piletsky, Trends Biotechnol. 2010, 28, 629; b) K. Haupt, Nat Mater 2010, 9, 612; c) A. Poma, M. J. Whitcombe, S. Piletsky, in Designing Receptors for the Next Generation of Biosensors, (Eds: S. Piletsky, M. J. Whitcombe), Springer-Verlag Berlin Heidelberg, Berlin 2013, Ch. 6.

[13] a) Y. Hoshino, R. C. Ohashi, Y. Miura, Adv. Mater. 2014, 26, 3718; b) P. Çakir, A. Cutivet, M. Resmini, B. T. S. Bui, K. Haupt, Adv. Mater. 2013, 25, 1048; c) Z. Chen, L. Xu, Y. Liang, M. Zhao, Adv. Mater. 2010, 22, 1488; d) M. Bompart, Y. De Wilde, K. Haupt, Adv. Mater. 2010, 22, 2343.

[14] a) D. Lakshmi, M. Akbulut, P. K. Ivanova-Mitseva, M. J. Whitcombe, E. V. Piletska, K. Karim, O. Güven, S. A. Piletsky, Ind. Eng. Chem. Res. 2013, 52, 13910; b) M. J. Whitcombe, I. Chianella, L. Larcombe, S. A. Piletsky, J. Noble, R. Porter, A. Horgan, Chem. Soc. Rev. 2011, 40, 1547; c) T. Takeuchi, D. Fukuma, J. Matsui, Anal. Chem. 1998, 71, 285; d) I. Chianella, M. Lotierzo, S. A. Piletsky, I. E. Tothill, B. Chen, K. Karim, A. P. Turner, Anal. Chem. 2002, 74, 1288.

[15] a) T. Miyata, M. Jige, T. Nakaminami, T. Uragami, Proc Natl Acad Sci U S A., 2006, 103, 1190; b) J. L. Bowen, PhD Thesis, Cardiff University, 2011.

[16] a) W. Bai, N. A. Gariano, D. A. Spivak, J. Am. Chem. Soc. 2013, 135, 6977; b) W. Bai, D. A. Spivak, Angew. Chem. Int. Ed. 2014, 53, 2095.

[17] a) A. Poma, A. Guerreiro, S. Caygill, E. Moczko, S. Piletsky, RSC Advances 2014, 4, 4203; b) A. Poma, A. Guerreiro, M. J. Whitcombe, E. V. Piletska, A. P. F. Turner, S. A. Piletsky, Adv. Funct. Mater. 2013, 23, 2821.

[18] T. Ami, K. Fujimoto, ChemBioChem 2008, 9, 2071. 


\section{WILEY-VCH}

[19] M. N. Stojanovic, P. de Prada, D. W. Landry, J. Am. Chem. Soc. 2001, 123, 4928.

[20] a) Z. Zhu, C. Wu, H. Liu, Y. Zou, X. Zhang, H. Kang, C. J. Yang, W. Tan, Angew.

Chem. Int. Ed. 2010, 49, 1052; b) K. Kang, A. Sachan, M. Nilsen-Hamilton, P. Shrotriya, Langmuir 2011, 27, 14696; c) B. R. Baker, R. Y. Lai, M. S. Wood, E. H. Doctor, A. J. Heeger, K. W. Plaxco, J. Am. Chem. Soc. 2006, 128, 3138; d) M. N. Stojanovic, D. W. Landry, J. Am. Chem. Soc. 2002, 124, 9678; e) R. J. White, N. Phares, A. A. Lubin, Y. Xiao, K. W. Plaxco, Langmuir 2008, 24, 10513; f) B.-C. Yin, B.-C. Ye, H. Wang, Z. Zhu, W. Tan, Chem. Commun. 2012, 48, 1248.

[21] A. Poma, H. Brahmbhatt, J. K. Watts, N. W. Turner, Macromolecules 2014, DOI: 10.1021/ma501530c.

[22] D. Rother, T. Sen, D. East, I. J. Bruce, Nanomedicine (Lond) 2011, 6, 281.

[23] E. Moczko, A. Poma, A. Guerreiro, I. Perez de Vargas Sansalvador, S. Caygill, F. Canfarotta, M. J. Whitcombe, S. Piletsky, Nanoscale 2013, 5, 3733.

[24] a) Y. Hoshino, T. Kodama, Y. Okahata, K. J. Shea, J. Am. Chem. Soc. 2008, 130, 15242; b) Y. Hoshino, W. W. Haberaecker III, T. Kodama, Z. Zeng, Y. Okahata, K. J. Shea, J. Am. Chem. Soc., 2010, 132, 13648; c) Y. Hoshino, M. Nakamoto, Y. Miura, J. Am. Chem. Soc., 2012, 134, 15209; d) M. Nakamoto, Y. Hoshino, Y. Miura, Biomacromolecules, 2014, 15, 541; e) S. Ambrosini, S. Beyazit, K. Haupt, B. Tse Sum Bui, Chem. Commun. 2013, 49, 6746.

[25] a) A. Guerreiro, A. Poma, K. Karim, E. Moczko, J. Takarada, I. P. de VargasSansalvador, N. Turner, E. Piletska, C. S. de Magalhaes, N. Glazova, A. Serkova, A. Omelianova, S. Piletsky, Adv. Healthc. Mater. 2014, DOI: 10.1002/adhm.201300634; b) D. Gao, Z. Zhang, M. Wu, C. Xie, G. Guan, D. Wang, J. Am. Chem. Soc. 2007, 129, 7859.

[26] E. Moczko, A. Guerreiro, E. Piletska, S. Piletsky, Langmuir 2013, 29, 9891. 


\section{WILEY-VCH}

[27] a) A. Plewa, S.-I. Yusa, M. Szuwarzyński, K. Szczubiałka, Y. Morishima, M. Nowakowska, J. Med. Chem. 2012, 55, 8712; b) K. Wybrańska, W. Niemiec, K. Szczubiałka, M. Nowakowska, Y. Morishima, Chem. Mater. 2010, 22, 5392.

[28] N. W. Turner, X. Liu, S. A. Piletsky, V. Hlady, D. W. Britt, Biomacromolecules 2007, 8, 2781.

[29] P. Cekan, E. O. Jonsson, S. T. Sigurdsson, Nucleic Acids Res. 2009, 37, 3990.

[30] G. Li, O. Shimelis, X. Zhou, R. W. Giese, BioTechniques 2003, 34, 908.

[31] M. Reth, Nat. Immunol. 2013, 14, 765.

[32] a) Q. Shen, L. Xu, L. Zhao, D. Wu, Y. Fan, Y. Zhou, W.-H. OuYang, X. Xu, Z. Zhang, M. Song, T. Lee, M. A. Garcia, B. Xiong, S. Hou, H.-R. Tseng, X. Fang, Adv. Mater. 2013, 25, 2368; b) J. Li, C. Fan, H. Pei, J. Shi, Q. Huang, Adv. Mater. 2013, 25, 4386; c) X. Yang, X. Liu, Z. Liu, F. Pu, J. Ren, X. Qu, Adv. Mater. 2012, 24, 2890; d) M. A. Nakatsuka, R. F. Mattrey, S. C. Esener, J. N. Cha, A. P. Goodwin, Adv. Mater. 2012, 24, 6010. 


\section{WILEY-VCH}

Here we present a strategy to exploit aptamers as recognition elements of molecularly imprinted polymeric nanoparticles (AptaMIP NPs), via a modification of the chemical structure of the DNA. We demonstrate that the introduction of this modified "aptamer monomer" results in an increase of the affinity of the produced MIP NPs, without altering their physical properties such as size, shape or dispersibility.

Molecularly imprinted polymers, nanoparticles, aptamers, solid-phase synthesis, cocaine

Alessandro Poma,* Heli Brahmbhatt, Hannah M. Pendergraff, Jonathan K. Watts, and Nicholas W. Turner*

Generation of Novel Hybrid Aptamer-Molecularly Imprinted Polymeric Nanoparticles (AptaMIP NPs)

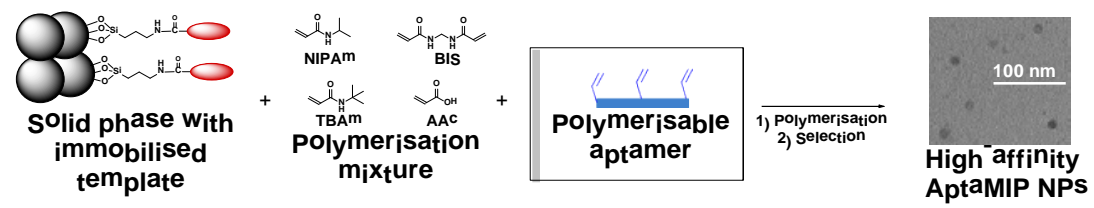




\section{WILEY-VCH}

Copyright WILEY-VCH Verlag GmbH \& Co. KGaA, 69469 Weinheim, Germany, 2013.

\section{Supporting Information}

Generation of Novel Hybrid Aptamer-Molecularly Imprinted Polymeric Nanoparticles (AptaMIP NPs)

Alessandro Poma,* Heli Brahmbhatt, Hannah M. Pendergraff, Jonathan K. Watts, and Nicholas W. Turner*

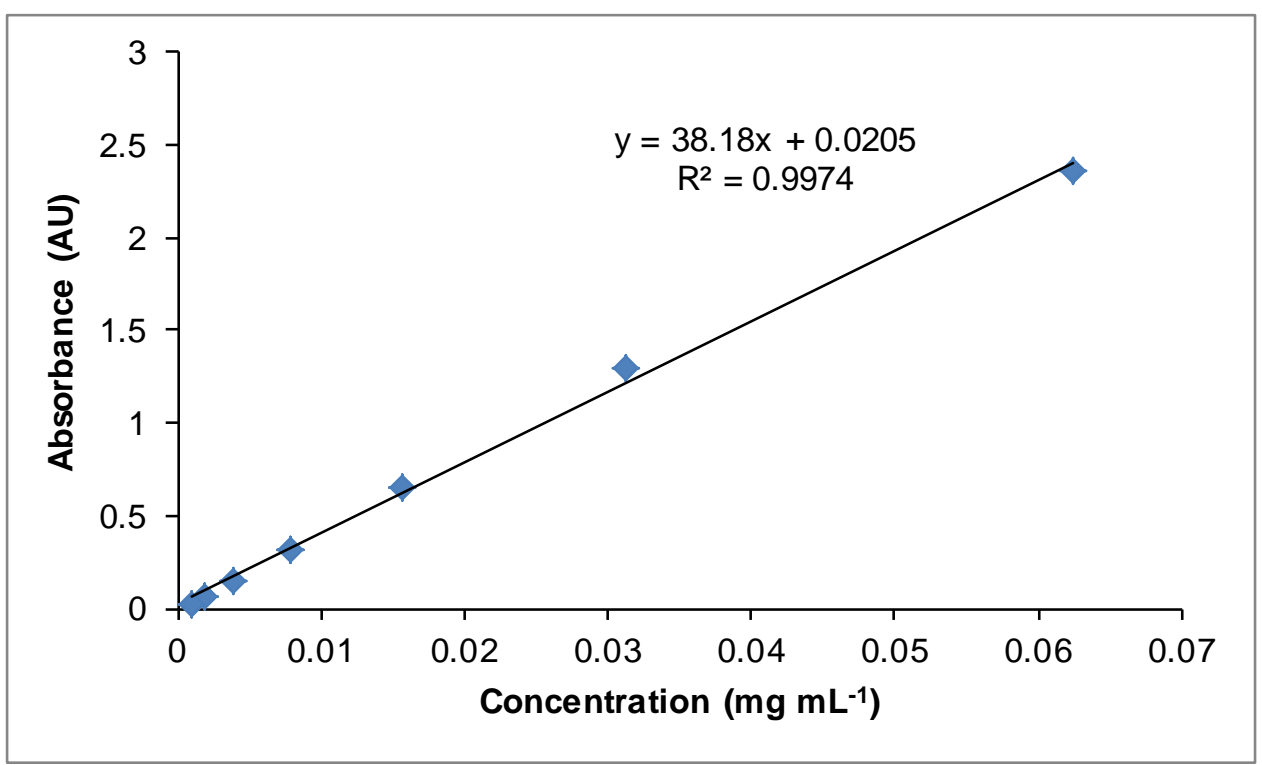

Figure S1. Calibration curve for the BEC template used to quantify the amount immobilized onto the glass beads. The concentrations range from $1.0 \times 10^{-3}$ to $6.25 \times 10^{-2} \mathrm{mg} \mathrm{mL}^{-1}$. The $\lambda_{\max }$ value used was $231 \mathrm{~nm}$.

Table S1 Static water contact angle measurements for surface-modified QCM crystals $(n=3)$.

\begin{tabular}{cc}
\hline Surface & Contact angle (degrees, \pm \\
SD)
\end{tabular}




\section{WILEY-VCH}

Table S2 Yields and dynamic light scattering (DLS) measurements for Plain, PolyT and AptaMIP NPs $(n=3)$.

\begin{tabular}{ccc}
\hline NPs & Yield (\%, w/w) & Diameter (nm, \pm SD) \\
\hline Plain MIP & $34 \%$ & $19 \pm 5$ \\
PolyT MIP & $36 \%$ & $17 \pm 1$ \\
AptaMIP Acrydite $^{\mathrm{TM}}$ & $40 \%$ & $15 \pm 1$ \\
AptaMIP T*1 $^{*}$ & $35 \%$ & $14 \pm 2$ \\
AptaMIP T*6 $^{*}$ & $39 \%$ & $19 \pm 4$ \\
\hline
\end{tabular}

